\title{
Particulate Formation During High Explosive Detonations: How Oxygen Balance Drives Parameters Pertinent to Atmospheric Lofting
}

Rachel C. Huber ( $\nabla$ rhuber@lanl.gov )

Los Alamos National Laboratory

Allison C. Aiken

Los Alamos National Laboratory

Dana M. Dattelbaum

Los Alamos National Laboratory

Manvendra K. Dubey

Los Alamos National Laboratory

Kyle Gorkowski

Los Alamos National Laboratory

David W. Podlesak

Los Alamos National Laboratory

\section{Research Article}

Keywords: High explosive (HE), oxygen, Composition B-3, PBX 9501, black carbon (BC), organic carbon (OC), von Neumann (VN)

Posted Date: October 8th, 2021

DOI: https://doi.org/10.21203/rs.3.rs-951616/v1

License: (c) (i) This work is licensed under a Creative Commons Attribution 4.0 International License.

Read Full License 


\title{
Particulate Formation During High Explosive Detonations: How Oxygen Balance Drives Parameters Pertinent to Atmospheric Lofting
}

\author{
Rachel C. Huber ${ }^{1, *}$, Allison C. Aiken ${ }^{2}$, Dana M. Dattelbaum ${ }^{1}$, Manvendra K. Dubey ${ }^{2}$, Kyle \\ Gorkowski ${ }^{2}$, and David W. Podlesak ${ }^{3}$
}

${ }^{1}$ Explosive Science and Shock Physics Division, Los Alamos National Laboratory, Los Alamos, NM, 87545, USA

${ }^{2}$ Earth and Environmental Science Division, Los Alamos National Laboratory, Los Alamos, NM, 87545, USA

${ }^{3}$ Chemistry Division, Los Alamos National Laboratory, Los Alamos, NM, 87545, USA

*rhuber@lanl.gov

LA-UR-21-29162

\begin{abstract}
High explosive (HE) detonations reach pressures and temperatures that extend beyond normal environmental conditions, thereby permitting access to various carbon and metal allotropes of different morphologies, sizes and surface structures. The products of $\mathrm{HE}$ detonations are dependent on multiple parameters, including the chemical and physical properties of the starting material and atmospheric conditions (i.e. oxygen). One important factor is the HE oxygen balance, which is the extent to which the material can be oxidized. Insensitive HEs are designed to resist external stimuli that would cause detonation in conventional HEs. The insensitive HEs are negatively oxygen balanced and therefore produce not only gaseous species but solid carbon products during detonation. Insensitive HEs were studied, Composition B-3 and PBX 9501, with steady and overdriven geometries in an oxygen-free atmosphere that reached different pressure and temperature regimes. Small angle $\mathrm{x}$-ray scattering provided the size and surface structure of the resulting particulates. Composition B-3 primary particles were $157.0 \pm 0.3 \AA$ and $199.5 \pm 0.3 \AA$ for steady and overdriven detonations; where PBX 9501 primary particles were larger than Composition B-3 at $300 \pm 6 \AA$ and $334.5 \pm 0.3 \AA$ for steady and overdriven detonations. The two compounds formed contrasting primary particles with different cluster structures, in the Composition B-3 steady detonation the particles were agglomerated into a surface fractal with rough surfaces where as the PBX 9501 was a mass fractal cluster with smooth surface primary particles. In the overdriven detonation the primary particles were reversed, Composition B-3 was agglomerated into a mass fractal structure with smooth surfaces and PBX 9501 had a surface fractal structure with a rough surface primary particles. Scanning electron microscopy provided a snapshot of the morphology of the materials on the micron length scale, supporting the observation of $x$-ray scattering that the Composition B-3 particulates/agglomerates are smaller than the PBX 9501. Raman spectroscopy provided information as to the carbon bonding of the detonation soot, showing significantly more product variation in Composition B-3 than PBX 9501, likely due the poor oxygen balance of Composition B-3 leading to more complex carbon bonding formations. Finally, $x$-ray photoelectron spectroscopy showed how the difference in the oxygen balance of the HE fuel directly relates to the amount of carbon-oxygen bonding that is present in the final products, where PBX 9501 had significantly more oxygen on the surface of the particulates. We used two HEs to understand the detonation pathways for both synthesis and atmospheric processes; where the chemical constituents of the particulates can promote processes such as self-lofting and aerosol-cloud interactions after the particles are launched into the troposphere or stratosphere during detonation.
\end{abstract}

\section{Introduction}

Aerosols emitted by fires, fossil combustion and high explosive (HE) detonations include black carbon (BC) particles, commonly referred to as soot, that significantly effect tropospheric and stratospheric chemistry, the earth's radiation balance, climate and air quality. ${ }^{1,2}$ Wildfire plumes can be approximated as $2 \% \mathrm{BC}$ and $98 \%$ organic carbon (OC) and can be lofted high by pyrocumulonimbus clouds. ${ }^{3}$ Submicron BC particulates are refractory, long lived and absorb sunlight allowing then to rise by solar heating, at a rate that depends on their properties. This is in contrast to OC that is more volatile and reactive making its atmospheric lifetime shorter due to evaporation and photooxidation. ${ }^{3,4}$ It has been recently observed that a $2 \%$ BC plume can rise to and remain in the stratosphere for many months, instead of atmospheric lifetimes on the order of days to weeks in the troposphere. ${ }^{3}$ Within the troposphere $\mathrm{BC}$ from biomass burning warms the air locally on a regional scale, but if it is lofted high in the troposphere or stratosphere it can cool the surface by blocking sunlight. ${ }^{5,6} \mathrm{BC}$ production from fires and fossil combustion at ambient pressures and temperatures $<1000 \mathrm{C}$ has been extensively studied and is well understood, for air quality 
and climate. ${ }^{7-9} \mathrm{BC}$ production from high temperature and high pressure $\mathrm{HE}$ detonations is nascent. There is a need to bridge the two regimes to assess transport of smoke and impacts of large HE detonations and subsequent fires. ${ }^{10-13}$ We analyze BC produced by two HEs with distinct pathways to gain mechanistic insights into the BC properties.

HE materials undergo a violent exothermic reaction when initiated, accessing extreme pressures $(P)$ and temperatures $(T)$. The specific $P$ and $T$ are dependent on the chemical and physical parameters of the HE. Chemically, energy is stored within the strained chemical structures, such as those in Figure 1A and B. ${ }^{14}$ Physically, the grain size, shape and smoothness of the HE grains also contribute to the effectiveness of the detonation. ${ }^{15}$ When the HE is ignited, the leading edge of the shockwave or von Neumann (VN) spike is the highest pressure the HE will reach as shown in Figure 1C. ${ }^{16}$ Between the VN spike and the Chapman-Jouguet (CJ) state is the Chemical Reaction Zone (CRZ). The CRZ is where the HE becomes a dense fluid of solid carbon and gases; where the CJ state is the pressure specific to the chemical equilibrium at the end of the CRZ. The Taylor wave is then the relaxation of the detonation back to atmospheric $P$ and $T$. Each HE behaves differently as the detonation proceeds in regards to product kinetics and formation, as authors have previously observed with Time-Resolved Small Angle X-ray Scattering (TRSAXS). ${ }^{17-21}$ By analyzing the post detonation soot, we can begin to untangle how certain HEs produce various carbon species. For example, nanodiamond (ND) is a well-known product from Composition B-3 (Comp B-3, 40\% TNT/60\% RDX) detonations. ${ }^{22}$ ND has been implemented in various scientific areas including medical application as biomarkers ${ }^{23}$ and thickeners in mechanical lubricants. ${ }^{24}$ Comp B-3 detonations have also been shown to produce graphitic structures and $s p^{2} / s p^{3}$ compounds that were dependent upon the detonation atmosphere. ${ }^{25}$ Other HEs, such as DNTF (3,4-bis(3-nitrofurazan-4-yl)furoxan)) reach $P$ and $T$ high enough to produce liquid carbon thereby synthesizing nano-onions ${ }^{26}$ An extensive amount of work has been performed to understand Comp B-3 detonation products (specifically ND), ${ }^{22,27,28}$ however the literature is sparse for other HEs and the resultant carbon allotropes. ${ }^{29}$
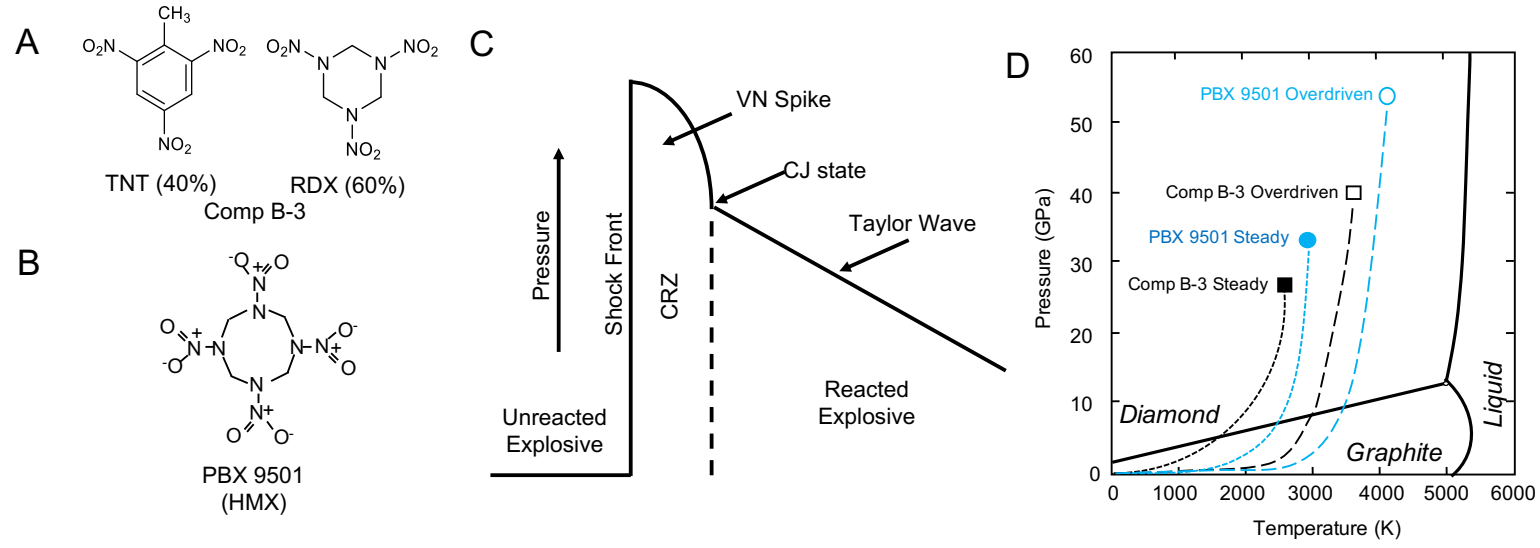

Figure 1. Chemical composition of Comp B-3 is $40 \%$ TNT and 60\% RDX (A) and PBX 9501 is composed of HMX in (B). C) Diagram showing how a detonation shock front moves through a material, including the VN spike, CJ state and CRZ (Figure reproduced from Deal ${ }^{30}$ ). D) Carbon phase diagram showing the CJ and overdriven states of Comp B-3 and PBX 9501. Comp B-3 is a lower $P / T$ HE than PBX 9501. At the CJ state both explosives reach $P$ and $T$ capable of producing diamond products, however only Composition B-3 is known to produce diamond species. The overdriven data point were determine through modeling, therefore the temperatures are not known.

In this work, we compared two different detonation geometries: 1) steady and 2) overdriven for both Comp B-3 (Figure 1A) and polymer bonded explosive (PBX) 9501 (HMX, octogen, Figure 1B). A steady detonation is a single shockwave moving uniaxially at supersonic speeds, where an overdriven detonation is produced by the collision of two steady detonations. For steady detonations of Comp B-3 and PBX 9501 the $P$ reaches $\sim 28 \mathrm{GPa}^{31}$ at $2800 \mathrm{~K}$ and $\sim 35 \mathrm{GPa}^{32}$ and $3000 \mathrm{~K}$. The overdriven detonations reach $P$ of $\sim 40$ and $\sim 54 \mathrm{GPa}$ for Comp B-3 and PBX 9501 according to calculations performed in Cheetah since overdriven pressures are dependent upon the input (be it colliding waves or a plate impact experiment). ${ }^{33}$ In Figure 1D, the carbon phase diagram shows the boundaries for graphite, diamond and liquid carbon. ${ }^{34}$ The CJ points for both HEs are plotted on the phase diagram for the steady detonations, where the reaction proceeds through the graphite boundary and into the diamond region. Comp B-3 is known to form ND ${ }^{22}$ and other high-order carbon species; however, PBX 9501 has not been as thoroughly explored. In this paper we will explore carbon and metal product formation from both steady and overdriven detonation geometries for Comp B-3 and PBX 9501 in a purposely inert atmosphere.

Previous work exploring Comp B-3 post detonation soot has shown that the surrounding atmosphere plays a vital role during carbon allotrope formation. ${ }^{25}$ That paper reported that detonations performed in an air atmosphere versus an argon atmosphere 
resulted in different carbon allotropes, which implies that the intercalation of oxygen microseconds into the detonation informed the carbon synthesis pathway. In this paper, we will explore Comp B-3 and PBX 9501 detonations all performed in an argon, or inert, atmosphere. By performing detonations in an inert atmosphere we negate long time intercalation of oxygen, thereby removing a parameter that would affect the synthesis pathway. The main difference between these HEs becomes the structure (Fig. 1A and B) and, therefore, the oxygen balance. The oxygen balance (OB) equation is

$$
O B \%=\frac{-1600}{M W}\left(2 C-\frac{H}{2}-O\right)
$$

where MW refers to the molecular weight and the $\mathrm{C}, \mathrm{H}$ and $\mathrm{O}$ refers to the number of the carbon, hydrogen and oxygen atoms. For Comp B-3 the chemical structure combines $60 \%$ RDX, $\mathrm{C}_{3} \mathrm{H}_{6} \mathrm{~N}_{6} \mathrm{O}_{6}$, and $40 \% \mathrm{TNT}, \mathrm{C}_{7} \mathrm{H}_{5} \mathrm{~N}_{3} \mathrm{O}_{6}$, resulting in an OB\% of $-42.6 \%$. PBX 9501, $\mathrm{C}_{4} \mathrm{H}_{8} \mathrm{~N}_{8} \mathrm{O}_{8}$, has an $\mathrm{OB} \%$ of $-21.6 \%$. An OB of zero would suggest that the molecule is perfectly balanced and only gas species would form during detonation. In this paper we will delve into a multi-length scale approach (scattering, microscopy, spectroscopy) to study the post detonation soot from Comp B-3 and PBX 9501 detonations performed in an inert atmosphere to better understand the chemistry behind $\mathrm{OB}$ and $P / T$ regimes.

\section{Results}

Post detonation soot was analyzed from HE detonations of Comp B-3 and PBX 9501 performed in inert atmosphere with small angle X-ray scattering (SAXS), scanning electron microscopy (SEM), Raman spectroscopy and X-ray photoelectron spectroscopy (XPS). These techniques provided bulk (SAXS and Raman) and surface (SEM and XPS) measurements to better understand how the chemical structure, $\mathrm{OB}$ and $P / T$ affects product formation.

\section{Small angle x-ray scattering}

SAXS is an ideal nondestructive method to analyze HE post detonation soot, providing the ability to determine the particle morphology, size and surface structure without typical harsh acid baths or oxidation. ${ }^{27,35}$ Representative SAXS patterns collected on post detonation soot from Comp B-3 steady and overdriven detonations are shown in Figure 2. Through both empirical and analytical methods, characteristic parameters can be construed from the scattering data. The SAXS patterns for each detonation geometry have a modest Guinier "knee", which implies that a majority of the carbon particles are larger than this scattering window, agglomerated or highly polydisperse. The radially-integrated SAXS patterns were analyzed with the Porod-Guinier unified fit approach, performed within Igor with the Irena software package. ${ }^{36}$ The unified fit allows for multiple length scales to be modeled within the SAXS data. In this study we used one Porod-Guinier region with a second low- $q$ Porod region because the data did not plateau towards low- $q$ for a second Guinier region. The unified function is

$$
\left.I(q)=\sum_{i=1}^{n}\left(G_{i} \exp \left(\frac{-q^{2} R_{g_{i}}^{2}}{3}\right)+\right)+B_{i} \exp \left(\frac{-q^{2} R_{g_{i+1}}^{2}}{3}\right) \times\left(q^{-1} \operatorname{erf}\left(\frac{q R_{g_{i}}}{\sqrt{6}}\right)^{3}\right)^{P_{i}}\right)
$$

where $R_{\mathrm{g}}$ is the radius of gyration, $G$ is the Guinier scaling factor, $B$ is the Porod scaling factor and $P$ is the Porod exponent (also known as $\alpha$ from fitting to the exponential $\mathrm{I}(q)=q^{-\alpha}$ ). As mentioned, we fit two levels where $i=1$ or 2 , level 1 being a Porod-Guinier and level 2 being only a Porod region. The $R_{\mathrm{g}}$ is the effective size of the system, with an overall assumption of a spherical particle. The Guinier "knee" and Porod exponents are graphed in Figure 2 . The $R_{\mathrm{g}}$ for Comp B-3 steady was $157.0 \pm$ $0.3 \AA$ and for overdriven was $199.5 \pm 0.3 \AA$.

Porod exponents are, in general, related to the electron density differences between the particle and the surrounding medium. A Porod exponent of -4 , for example, suggests a smooth surface and a distinct difference in electron density between the particle and its surroundings. If an exponent of -3 is found, that suggests a surface fractal, -2 is platelets or flattened structures and -1 is represenative of a rod structure. ${ }^{37,38}$ Porod exponents that are non-integers are described as intermediates of the integer exponents. For example, glassy carbon has a surface fractal structure with a Porod exponent of $-3.2 .^{39,40}$ In Figure 2 , the high- $q$ Porod exponents correspond to the individual particulates surface structure. ${ }^{41}$ In the case of Comp B-3 steady and overdriven Porod exponents were -3.6 and -4.0 in concurrance with the unified fit. The steady detonation corresponds with a surface fractal as similarly fit to carbon black soots. ${ }^{42}$ The overdriven detonation shows a distinct difference between the particles and the surround medium with the Porod slope of -4.0 .

Now, the low- $q$ Porod exponents, in Figure 2, corresponds to either larger particles or, as likely in this case, the agglomeration of the low $-q$ particulates. The exponents for the steady and overdriven detonations were -3.0 and -2.7 . As mentioned above, the -3.0 exponent corresponds to a surface fractal, where the -2.7 slope would correspond to a mass fractal, with -2.5 often observed as a Porod exponent for that mass fractal agglomeration of carbon black soot. ${ }^{42}$ The combination of the high- $q$ and low- $q$ Porod exponents suggests that the steady detonations produce particulates that have rough surfaces and are agglomerated in a fractal like structure. For overdriven Comp B-3 detonations the particles have smooth surfaces and are aggregated like a mass fractal. 

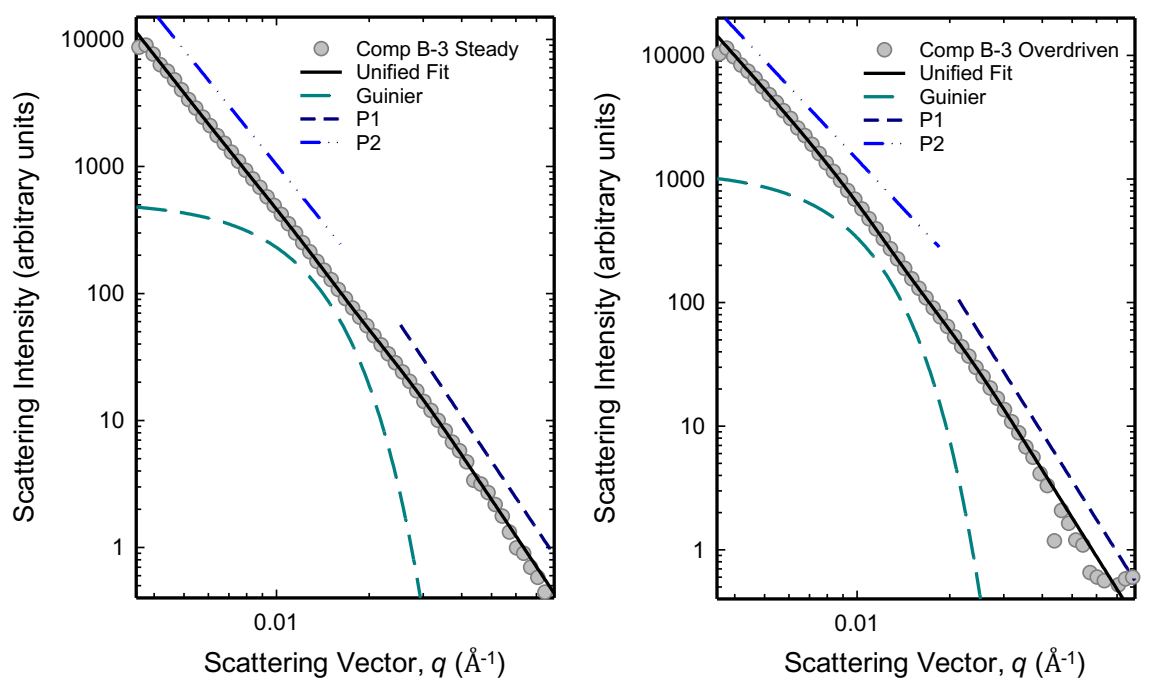

Figure 2. Radially-integrated SAXS patterns for Comp B-3 steady and overdriven detonations. A) Comp B-3 steady detonation was fit to a power law slope at high- $q$ of -3.6 and at low- $q$ of -3.0 , indicative of a surface fractals for both the primary particle and the agglomeration of those particles. The unified fit, which includes the Guinier region, resulted in a $R_{\mathrm{g}}$ of $157 \pm 0.25 \AA$ A. B) Comp B-3 overdriven detonation fit to a power law slope at high- $q$ of -4.0 and low- $q$ of -2.7 , suggestive of smooth surface agglomerated into a mass fractal, respectively. The unified fit produced a $R_{\mathrm{g}}$ of $199.5 \pm 0.3 \AA$.

PBX 9501, as discussed in the Introduction, differs in its oxygen balance compared to Comp B-3, producing particulates of different sizes and surface structures (Figure 3). The PBX 9501 unified fit resulted in a $R_{\mathrm{g}}$ of $300 \pm 6 \AA$ and $334.5 \pm 0.3 \AA$ for steady and overdriven detonations. The PBX 9501 particulate sizes are larger than the Comp B-3 particulates, likely due to the Comp B-3 lower $P$ and $T$ reached during detonation that resulted in the quenching of particle growth ("freezing out") happening quicker than in the PBX 9501. ${ }^{20}$ As for the high- $q$ Porod exponents, the steady and overdriven values were -4.2 and -3.7. This suggests a smooth surface with a distinct difference between materials for the steady detonation particles and a surface fractal structure for the overdriven particulates. The low- $q$ Porod exponents for PBX 9501 steady and overdriven detonations were -2.5 and -3.5 , respectively. These Porod exponents suggests both mass and surface fractal aggregation for the steady and overdriven detonations, respectively. The combination of the Porod exponents for the steady detonation of PBX 9501 indicates that the particles have a smooth surface and are agglomerated into a mass fractal structure; where the overdriven detonation produced particles with rough surfaces arranged into a surface fractal.

\section{Scanning electron microscopy}

The SEM images in Figure 4 show the particles from each of the detonations with a scale bar of $5 \mu \mathrm{m}$. The lighter colored particles are $\mathrm{Cu}$ or $\mathrm{Zn}$ particles from the brass detonators used for these detonations. The lighter gray particles are aluminum, which are from the container that the detonations were performed in. The darker gray particles are the carbon materials. In all of the detonations there is a large amount of metal contaminants, all with a relatively spherical conformation. The carbon particles are often where we observe a variation in the morphology. For example, in Figure 4A and B, which were steady and overdriven Comp B-3 detonations that show platelet-like carbon particles (more so in the overdriven case than the steady). Or in Figure $4 \mathrm{C}$ and $\mathrm{D}$, where it is obvious that the metal particles are coated with carbon (the light gray, almost airy looking material). The largest particulates for the Comp B-3 steady and overdriven detonations were $0.87 \mu \mathrm{m}$ and $0.92 \mu \mathrm{m}$, with the average particle size being $0.37 \mu \mathrm{m}$ and $0.20 \mu \mathrm{m}$. The PBX 9501 detonations produced larger particles than the Comp B-3 detonations, with the largest particles measuring $1.18 \mu \mathrm{m}$ and $2.38 \mu \mathrm{m}$ for the steady and overdriven detonations. The average particles sizes for PBX 9501 for steady and overdriven detonations were $0.27 \mu \mathrm{m}$ and $0.13 \mu \mathrm{m}$. The particulate sizes produced from these detonations are heterogenous due to the extreme nature of the synthesis process. Overall, the particles from the Comp B-3 detonations are smaller than those from the PBX 9501 detonations, which is similar to what was observed in the SAXS section, although on a micrometer length scale. 


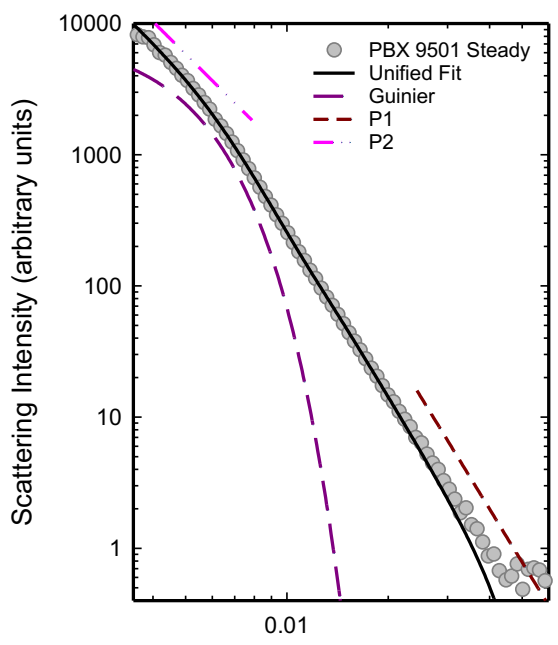

Scattering Vector, q (A-1)

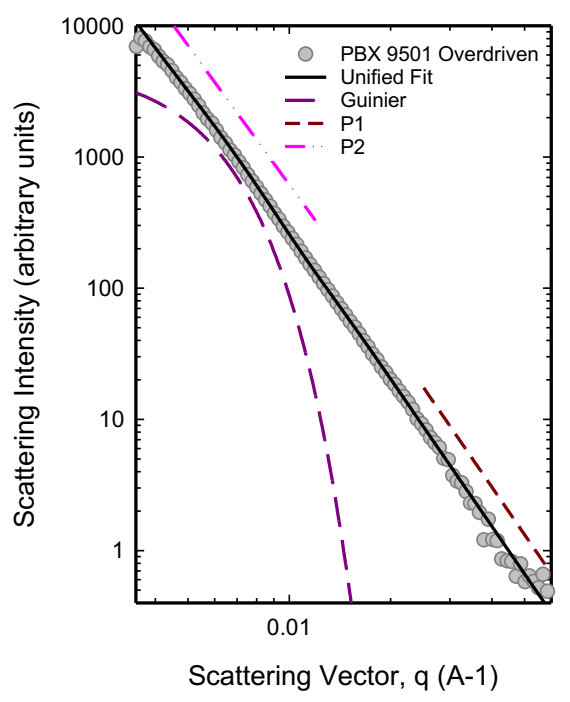

Figure 3. Radially-integrated SAXS patterns from PBX 9501 steady and overdriven detonations. A) The PBX 9501 steady detonation at high- $q$ had a Porod exponent of -4.2 and low- $q$ of -2.5 , indicative of primary particles with smooth surfaces agglomerated into a mass fractal-like structure. The unified fit produced a $R_{\mathrm{g}}$ of $300 \pm 6 \AA$. B) The PBX 9501 overdriven detonation gave a Porod exponent at high- $q$ of -3.7 , with a low- $q$ exponent of -3.5 . This suggests, in general, that the particles have rough surfaces and are agglomerated in a fractal-like structure. The unified fit resulted in a $R_{\mathrm{g}}$ of $334.5 \pm 0.3 \AA$.

\section{Spectroscopy}

\section{Raman spectroscopy}

We used Raman spectroscopy to dive deeper into the previous physical observation, to understand how the previous larger scale material properties relate to the carbon bonding. Raman spectroscopy is a bulk scattering method that provides information about the carbon bonding of the post detonation soot without harsh chemical separation. Figure 5 plots the Raman peak positions against the full width at half maximum (FWHM), with the values reported in Table 1. Soot samples herein were fit in accordance with previously published procedures. ${ }^{43}$ These Raman spectra contained two main peaks, G and D, with two underlying peaks labeled as D4 and D3. ${ }^{43}$ Figure 4A contains the data for Comp B-3 steady and overdriven detonations. The main peak in these spectra is the D peak, or $s p^{2}$ defect peak present at $1296 \pm 4 \mathrm{~cm}^{-1}$ and $1314 \pm 4 \mathrm{~cm}^{-1}$ for steady and overdriven detonations. ${ }^{44}$ The next largest peak is the $\mathrm{G}$ peak, which is the first order $s p^{2}$-allowed Raman peak, found at 1581 $\pm 5 \mathrm{~cm}^{-1}$ and $1587 \pm 2 \mathrm{~cm}^{-1}$ for steady and overdriven soot. ${ }^{43}$ Disordered carbon species are denoted by the D4 peak, present at $1121 \pm 20 \mathrm{~cm}^{-1}$ and $1204 \pm 16 \mathrm{~cm}^{-1}$ for steady and overdriven detonations. ${ }^{45}$ Finally, the amorphous carbon peak, D3, is present in both steady and overdriven detonations at $1450 \pm 10 \mathrm{~cm}^{-1}$ and $1485 \pm 8 \mathrm{~cm}^{-1}$. ${ }^{46}$ This peak is fit underneath and between the D and G peaks. All of the Comp B-3 overdriven Raman peaks are shifted to higher wavenumbers than the steady detonation soot. The highest deviation with regards to peak positions exists within the D3 and D4 peak due to these peaks representing various carbon species that are not just simple graphite but different carbon bonding defects.

PBX 9501 peaks show far less scatter than the Comp B-3 peaks, when comparing A and B in Fig. 5. Each data point for D4, D, D3 and G all appear relatively close to within error. The graphite D peak appears at $1300 \pm 3$ and $1306 \pm 4 \mathrm{~cm}^{-1}$ for steady and overdriven detonations. The $\mathrm{G}$ peak is also similar for steady and overdriven detonations at $1496 \pm 11$ and $1504 \pm$ $16 \mathrm{~cm}^{-1}$. Even the defect, D4, peak and amorphous, D3, peak fit to very similar peak positions, as shown in Table 1 and in Figure 5B. This Raman data for Comp B-3 and PBX 9501 implies that the steady and overdriven detonation processes have more affect on the Comp B-3 particulate formation synthetically than it does on the PBX 9501, likely due to the effect of HE oxygen balance.

\section{$X$-ray photoelectron spectroscopy}

XPS provides surface structure information between the steady and overdriven Comp B-3 and PBX 9501 detonations. The XPS spectra were fit in agreement with previously published literature, the results are found in Table $2 .{ }^{47}$ The carbon and oxygen peaks were scanned at high resolution to obtain the carbon and oxygen atomic ratios. Comparing the overall ratios between Comp B-3 and PBX 9501 automatically shows a distinct difference due to starting material. The carbon and oxygen atomic ratios for Comp B-3 are 95:5 and 94:6 for steady and overdriven detonations. As we presented in a previous paper, Comp B-3 

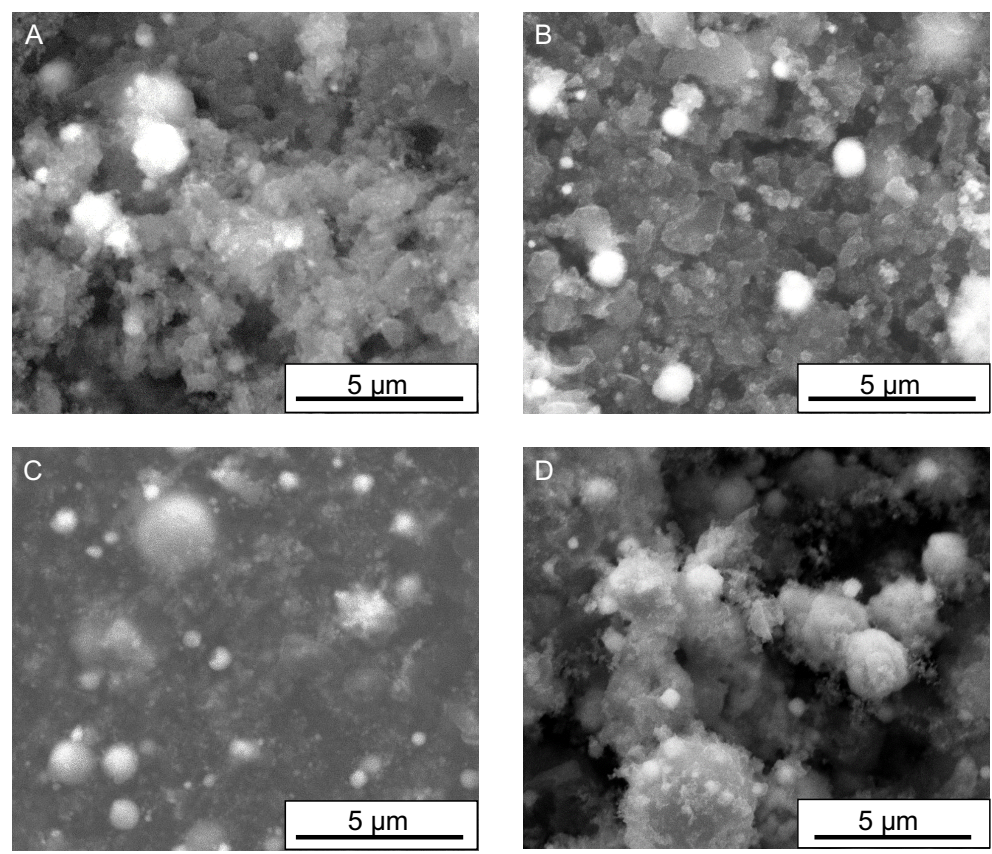

Figure 4. SEM images from Comp B-3 steady (A), overdriven (B), PBX 9501 steady (A) and overdriven (B) detonations. The white particulates are either pure $\mathrm{Cu}$ or $\mathrm{Zn}$ particles from the brass detonators used for the detonation initiation. The light gray particles are $\mathrm{Al}$ from the lining of the detonation chamber. Finally, the dark gray particles are carbon particles produced during the detonation from the HE.

steady detonation performed in an air atmosphere showed a carbon to oxygen ratio of 36:64, thereby demonstrating how an air atmosphere affects the surface structure of the soot particles. ${ }^{25}$ The carbon dominates the surface of these particles when a detonation is performed in an argon atmosphere, resulting in very little $\mathrm{O}$ termination at the surface.

Remarkably, the PBX 9501 shows carbon to oxygen atomic ratios of 62:38 and 76:24 for steady and overdriven detonations. The oxygen balance for PBX 9501, as mentioned in the introduction, is $-21.6 \%$, compared to Comp B-3 that is $-42.6 \%$. This suggests that more gas should be produced during detonation, however it also means that the oxygen that is unable to react to a gaseous species in the time allotted from the PBX 9501 detonation will integrate into the solid carbon products. This statement is obvious from the $\mathrm{C}: \mathrm{O}$ ratios determined through XPS. Interestingly, the overdriven detonation resulted in a higher amount of carbon in the soot. This is likely due to the overdriven detonation initially forming particulates before collision, then those particulates experience higher $P$ and $T$ during collision resulting in more gaseous products formed than during a steady detonation.

For determination of $s p^{2}$ and $s p^{3}$ percentages we fit the carbon peak to five separate peaks centered at $284,285,286$, 287 and $289 \mathrm{eV}$, as previously shown in literature. ${ }^{47,48} \mathrm{The} s p^{2}$ and $s p^{3}$ peaks are known to be centered at 284 and $285 \mathrm{eV}$, respectively. The other three peaks, 286,287 and $289 \mathrm{eV}$, fit the tail of the spectrum, which is theorized to be composed of various C-O compounds. ${ }^{47,48}$ The values for $s p^{2} \%$ and $s p^{3} \%$ are found in Table 2, interestingly these values are similar between all detonation geometries. The $s p^{2} \%$ values are mid $60 \%$, where the $s p^{3} \%$ values are $\sim 20 \%$. It is vital to mention that the $s p^{3} \%$ could encompasses both diamond and diamond-like carbon (DLC), where DLC is $s p^{3}$ amorphous carbon. ${ }^{49,50}$ In Comp $\mathrm{B}-3$ it is known that some percentage of the soot is nanodiamond, therefore it is unsurprising that the $s p^{3}$ peak is observed. ${ }^{22}$ However, nanodiamond has not been observed in PBX 9501 post detonation soot, that implies that the $s p^{3}$ observed in the case of PBX 9501 may actually be DLC.

\section{Discussion}

Understanding carbon product formation during high $P$, high $T$ HE detonations is not a trivial task. It requires knowledge of HE detonations and their pathways, from the VN spike to the CJ point and how steady and overdriven detonations change that pathway. How even though a HE may reach a similar $P$ and $T$ (such as Comp B-3 overdriven and PBX 9501 steady detonations), that may not result in the same products, just as we observed in this paper. The starting materials chemical constituents and morphology play a large role in how steady the shock front is, to how much chemical energy is stored within the strained rings. As we presented in this paper the products observed from detonations of Comp B-3 and PBX 9501 in an inert atmosphere are 


\begin{tabular}{lcccc}
\hline Detonation Condition & Peak & Peak Position $\left(\mathrm{cm}^{-1}\right)$ & Peak Height & FWHM $\left(\mathrm{cm}^{-1}\right)$ \\
\hline Comp B-3 Steady & D4 & $1121(20)$ & $0.16(4)$ & $159(61)$ \\
& D & $1296(4)$ & $0.92(6)$ & $165(22)$ \\
& D3 & $1450(10)$ & $0.33(6)$ & $147(58)$ \\
& G & $1581(5)$ & $0.51(5)$ & $108(16)$ \\
\hline Comp B-3 Overdriven & D4 & $1204(16)$ & $0.19(7)$ & $132(43)$ \\
& D & $1314(4)$ & $0.95(6)$ & $156(17)$ \\
& D3 & $1485(8)$ & $0.24(3)$ & $131(43)$ \\
& G & $1587(2)$ & $0.46(4)$ & $68(8)$ \\
\hline PBX 9501 Steady & D4 & $1162(15)$ & $0.11(3)$ & $106(50)$ \\
& D & $1300(3)$ & $0.98(2)$ & $152(10)$ \\
& D3 & $1496(11)$ & $0.18(2)$ & $125(48)$ \\
& G & $1592(2)$ & $0.45(4)$ & $60(9)$ \\
\hline PBX 9501 Overdriven & D4 & $1173(17)$ & $0.13(5)$ & $109(62)$ \\
& D & $1306(4)$ & $0.96(3)$ & $164(16)$ \\
& D3 & $1504(16)$ & $0.19(4)$ & $134(66)$ \\
& G & $1596(3)$ & $0.46(5)$ & $61(12)$ \\
\hline
\end{tabular}

Table 1. Raman spectroscopy performed on post detonation Comp B-3 and PBX 9501 samples for steady and overdriven detonations. The table contains the D4, D, D3 and G peaks that relate to carbon species within the sample denoting the peak position, peak height and FWHM. () denotes the error in the last digit.

\begin{tabular}{lcccc}
\hline Detonation Condition & C Atomic Ratio & O Atomic Ratio & $\mathrm{sp}^{2} \%$ & $\mathrm{sp}^{3} \%$ \\
\hline Comp B-3 Steady & 95 & 5 & 67 & 16 \\
\hline Comp B-3 Overdriven & 94 & 6 & 65 & 21 \\
\hline PBX 9501 Steady & 62 & 38 & 62 & 17 \\
\hline PBX 9501 Overdriven & 76 & 24 & 67 & 19 \\
\hline
\end{tabular}

Table 2. XPS data for Comp B-3 and PBX 9501 steady and overdriven detonations. The atomic $\mathrm{C}$ and $\mathrm{O}$ ratios were obtained through high resolution scans of the $\mathrm{C}$ and $\mathrm{O}$ peaks. The $\mathrm{sp}^{2} \%$ and $\mathrm{sp}^{3} \%$ were determined by fitting the $\mathrm{sp}^{2}$ peak at $284 \mathrm{eV}$ and $\mathrm{sp}^{3}$ peak at $285 \mathrm{eV}$.

relatively different according to the bulk and surface measurements that were performed.

The unified fit from the SAXS data provided the $R_{\mathrm{g}}$ (effective size) of the particles within the detonation soot for steady and overdriven detonations of Comp B-3 and PBX 9501. The Comp B-3 $R_{\mathrm{g}}$ values were smaller than those fit for PBX 9501. From the modeling time-resolved small angle x-ray scattering (TRSAXS) for both PBX 9502 and Comp B-3 there is a "freeze out" temperature assumed for HE detonations; this is a temperature where the primary particles stop growing and agglomeration begins. ${ }^{20}$ It has been shown between steady and reflected (where the shockwave was reflected off a tantalum plate, producing an above CJ state) that this "freeze out" temperature ends up producing larger particles for higher pressure detonations, as we have shown here between Comp B-3 and PBX 9501. ${ }^{21}$ It is important to note that all of the TRSAXS experiments were performed under vacuum so these experiments do compare well with the inert atmosphere experiments performed here. Further, data fit from this TRSAXS data comparing PBX 9501, RDX and TNT showed that PBX 9501 produced larger particles than TNT, but not RDX; however, the averaging of those particulate sizes (RDX and TNT) would have resulted in particulates that were smaller than PBX 9501. ${ }^{51}$ Since the Comp B-3 overdriven and PBX 9501 steady detonations reached similar pressures (40 vs. $35 \mathrm{GPa}$ ) this also implies that the initial chemical structure of the HE helps direct product formation, or the particulates for Comp B-3 steady would be larger than the PBX 9501 overdriven detonation.

The difference between the graphitic structures in the Raman spectroscopy is most likely tied to the OB and therefore the efficiency of the detonation. Comp B-3 Raman peaks are not within error for the steady and overdriven detonation. With the poor $\mathrm{OB},-42.6 \%$, the majority of the $\mathrm{O}$ reacts to gaseous species. For the overdriven detonation the majority of the $\mathrm{O}$ has reacted to gas before the colliding waves met, as shown by XPS with a C:O ratio of 95:5 for the steady detonation. Once those steady waves meet there is a lack of oxygen to create gas species, therefore those carbon species experience the 40 GPa pressure increase. That increase in pressured tied to the pure carbon species, unsurprisingly results in variations between the graphitic peaks in the Raman scattering between the steady and overdriven Comp B-3 detonations. However, the PBX 9501 is better OB at $-21.6 \%$, providing more oxygen to mixture with the solid carbon and gas. The XPS C:O ratio of 62:38 was measured for the 

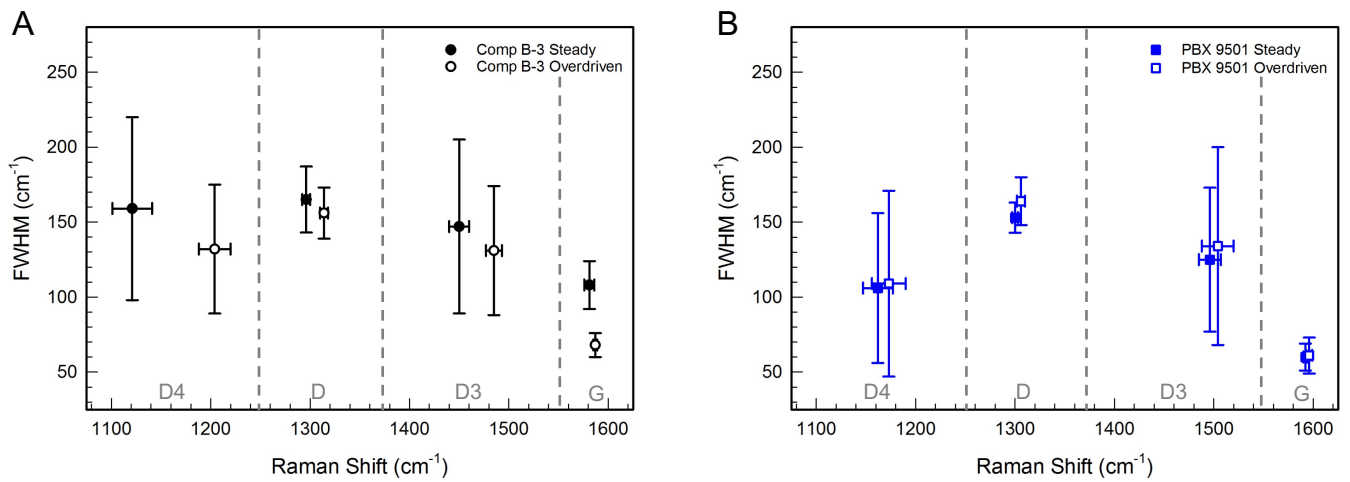

Figure 5. Peak position verse FWHM determine from Raman spectroscopy on Comp B-3 and PBX 9501 steady and overdriven detonations. The $\mathrm{D}$ and $\mathrm{G}$ peaks are the graphite defect peak and $s p^{2}$ first order allowed graphite peak, where the D4 and D3 peaks are the edge-defect peak and amorphous carbon peak. A) Comp B-3 steady and overdriven detonations show signifiant differences between the steady and overdriven peak positions implying variations between the products produced during the two detonations. B) PBX 9501 steady and overdriven post detonation soot Raman peaks show very little variation between the two detonation conditions.

steady detonation, therefore $\mathrm{O}$ was still present for the overdriven detonation when the two steady shockwaves collide and experience the increase in pressure.

These particulates formed during both steady and overdriven detonations did not fit to particle sizes larger than $\sim 300 \AA$ ( 30 $\mathrm{nm}$ ). Soot formation during Comp B-3 detonations produced a dark black soot, where PBX 9501 detonation produced light gray soot. It is likely that a Comp B-3 detonation performed in open-air would loft BC particles within the troposphere, if not up into the stratosphere, where these particles would linger for months perhaps affecting cloud formation or further heating the ozone layer. ${ }^{3,5}$ The SEM does also show both agglomeration of particulates and particles that are larger than those measured from the SAXS that would not be lofted as high thereby not contributing to the net radiative forcing effect. The resultant light colored PBX 9501 soot is supported by the C:O ratios discussed above, where the soot has significantly more oxygen than the Comp B-3 post detonation soot. Therefore it is likely that the PBX 9501 soot would react with the ozone or OH radicals if lofted to the stratosphere, thereby not lofting as high and having a shorter atmospheric lifetime in the stratosphere like Comp B-3 particulates.

This paper brings together bulk and surface scattering techniques to better understand how carbon species form during HE detonations. The more negative the $\mathrm{OB}$, the more variation was observed in the post detonation soot as observed for Comp B-3. PBX 9501, with an OB of $-21.6 \%$, produced particulates with more oxygen bonding and that were more similar between the steady and overdriven detonations than those produced during the detonation of Comp B-3.

\section{Methods}

\section{High Explosive Detonations}

HE Comp B-3 (lot \# HOL85G461-004, Holston) composed of 40\% trinitrotoluene (TNT), 60\% cyclotrimethylenetrinitramine (RDX: 6.5\% octogen HMX), and PBX 9501 (cyclotetramethylene-tetranitramine, lot \# HOL89C730-010) was warm-pressed into pellets weighing $\sim 2.7 \mathrm{~g}$, (L: $20 \mathrm{~mm}$; OD: $10 \mathrm{~mm}$; Density: $1.7 \mathrm{~g} / \mathrm{cm}^{3}$ ). Four pellets were epoxied together with Aralhex $\mathrm{B}$ (Los Alamos urethane-based adhesive) to create a detonation stick weighing $\sim 10.8 \mathrm{~g}$. Detonations were initiated using an RP-1 detonator (Reynolds). Detonations were conducted in a stainless steel cylinder, $(\mathrm{OD}=0.3 \mathrm{~m}$; Wall Thickness $=0.013 \mathrm{~m}$; Length $=0.61 \mathrm{~m}$; Aluminum caps: $0.356 \mathrm{~m} \times 0.356 \mathrm{~m}$ x $0.0254 \mathrm{~m}$ ) under Ar after a 10 minute purge with Ar. The amount of

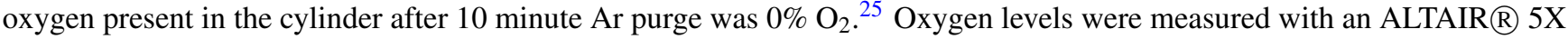
Gas Detector (MSA, PA). After detonation the solid residues were collected by vacuum onto an Ag filter.

\section{Small Angle X-ray Scattering}

Post detonation soot was analyzed in a transmission geometry at beamline 1-4 at Stanford Synchrotron Radiation Lightsource.

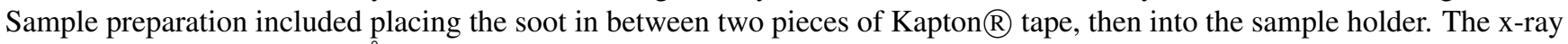
energy was $10 \mathrm{KeV}(\lambda=1.24 \AA)$. Each sample was collected for 300 seconds. The $2 \mathrm{D}$ images were radially-integrated resulting in the $1 \mathrm{D}$ data, with a $q$-range from $0.003-0.07 \AA^{-1}$. Kapton $\AA$ tape was run as a blank to account for any scattering that was not the post detonation soot. 


\section{Scanning electron microscopy}

SEM images were collected with a FEI Quanta 200 FEG SEM. The beam energy was $20 \mathrm{nA}$ and the accelerating voltage was $20 \mathrm{kV}$. The samples were collected on carbon sticky tape SEM studs that were grounded to the holder with copper tape during collection of images. Both backscattered electron (BSE) and Everhart-Thornley detector (ETD) images were captured, the ETD images are presented in this paper. The particles in the SEM images were analyzed within ImageJ with Analyze Particles. ${ }^{52}$

\section{Raman spectroscopy}

Raman spectroscopy was performed with an Invictus diode $785 \mathrm{~nm}$ laser (Kaiser Optical Systems, Inc., MI). Short acquisition times $(1 \mathrm{~s})$ were used to optimize the focal length, then longer times were used to reduce signal to noise $(20-60 \mathrm{~s}$, time varied between samples) and collect data. Data was collected on a cryogenically cooled charge coupled device detector (Princeton Instruments, $\mathrm{NJ}$ ). Resolution of the instrument is $5 \mathrm{~cm}^{-1}$.

\section{Photoelectron spectroscopy}

Solid carbon residues were characterized for amount of carbon and oxygen by X-ray photoelectron spectroscopy (XPS). XPS was performed using a Physical Electronics VersaProbe (ULVAC-PHI, MN). Soot collected on the Ag filter was directly inserted into the XPS for analysis. First a survey scan was performed, then a high resolution scan of the carbon and oxygen peaks was collected.

\section{References}

1. DeMott, P. J. et al. Predicting global atmospheric ice nuclei distributions and their impacts on climate. Proc. Natl. Acad. Sci. 107, 11217-11222, DOI: 10.1073/pnas.0910818107 (2010).

2. Boucher, O. et al. Climate change 2013: The physical science basis. contribution of working group i to the fifth assessment report of the intergovernmental panel on climate change. Report (2013).

3. Yu, P. et al. Black carbon lofts wildfire smoke high into the stratosphere to form a persistent plume. Science 365, 587-590 (2019).

4. Michelsen, H. A. et al. A review of terminology used to describe soot formation and evolution under combustion and pyrolytic conditions. ACS Nano 14, 12470-12490, DOI: 10.1021/acsnano.0c06226 (2020).

5. Fan, J. et al. Substantial convection and precipitation enhancements by ultrafine aerosol particles. Science 359, 411-418 (2018).

6. Bond, T. C. et al. Bounding the role of black carbon in the climate system: A scientific assessment. J. Geophys. Res. Atmospheres 118, 5380-5552, DOI: https://doi.org/10.1002/jgrd.50171 (2013). https://agupubs.onlinelibrary.wiley.com/ doi/pdf/10.1002/jgrd.50171.

7. Maricq, M. M. Examining the relationship between black carbon and soot in flames and engine exhaust. Aerosol Sci. Technol. 48, 620-629, DOI: 10.1080/02786826.2014.904961 (2014).

8. Michelsen, H. A. Probing soot formation, chemical and physical evolution, and oxidation: A review of in situ diagnostic techniques and needs. Proc. Combust. Inst. 36, 717-735, DOI: 10.1016/j.proci.2016.08.027 (2017).

9. Johansson, K. O., Head-Gordon, M. P., Schrader, P. E., Wilson, K. R. \& Michelsen, H. A. Resonance-stabilized hydrocarbonradical chain reactions may explain soot inception and growth. Science 361, 997-1000, DOI: 10.1126/science.aat3417 (2018). https://science.sciencemag.org/content/361/6406/997.full.pdf.

10. Liu, S. et al. Aerosol single scattering albedo dependence on biomass combustion efficiency: Laboratory and field studies. Geophys. Res. Lett. 41, 742-748, DOI: https://doi.org/10.1002/2013GL058392 (2014). https://agupubs.onlinelibrary.wiley. com/doi/pdf/10.1002/2013GL058392.

11. Saleh, R. et al. Brownness of organics in aerosols from biomass burning linked to their black carbon content. Nat. Geosci. 7, 647-650, DOI: 10.1038/ngeo2220 (2014).

12. China, S., Mazzoleni, C., Gorkowski, K., Aiken, A. C. \& Dubey, M. K. Morphology and mixing state of individual freshly emitted wildfire carbonaceous particles. Nat. Commun. 4, 2122, DOI: 10.1038/ncomms3122 (2013).

13. Johansson, K. O. et al. Formation and emission of large furans and oxygenated hydrocarbons from flames. Proc. Natl. Acad. Sci. 113, 8374-8379, DOI: 10.1073/pnas.1604772113 (2016). https://www.pnas.org/content/113/30/8374.full.pdf.

14. Tarver, C. Chemical energy release in self-sustaining detonation waves in condensed explosives. Combust. Flame 46, 157 176, DOI: https://doi.org/10.1016/0010-2180(82)90013-X (1982). 
15. Skidmore, C. B., Phillips, D. S., Howe, P. M., Mang, J. T. \& Romero, J. A. The evolution of microstructural changes in pressed hmx explosives. Eleventh Int. Detonation Symp. (1998).

16. Döring, W. Über den detonationsvorgang in gasen. Annalen der Physik 435, 421-436, DOI: 10.1002/andp.19434350605 (1943). https://onlinelibrary.wiley.com/doi/pdf/10.1002/andp.19434350605.

17. Bagge-Hansen, M. et al. Measurement of carbon condensates using small-angle $x$-ray scattering during detonation of the high explosive hexanitrostilbene. J. Appl. Phys. 117, 245902, DOI: 10.1063/1.4922866 (2015).

18. Firestone, M. A. et al. Structural evolution of detonation carbon in composition b-3 by x-ray scattering. AIP Conf. Proc. 1793, 030010 (2017).

19. Gustavsen, R. L. et al. Time resolved small angle x-ray scattering experiments performed on detonating explosives at the advanced photon source: Calculation of the time and distance between the detonation front and the x-ray beam. J. Appl. Phys. 121, 105902, DOI: 10.1063/1.4978036 (2017).

20. Watkins, E. B. et al. Evolution of carbon clusters in the detonation products of the triaminotrinitrobenzene (tatb)-based explosive pbx 9502. The J. Phys. Chem. C 121, 23129-23140, DOI: 10.1021/acs.jpcc.7b05637 (2017).

21. Huber, R. et al. Time resolved small angle $x$-ray scattering measurements of carbon coagulation for normal and reflected wave detonations. Sixt. Int. Detonation Symp. (2018).

22. Greiner, N. R., Phillips, D. S., Johnson, J. D. \& Volk, F. Diamonds in detonation soot. Nature 333, 440-442 (1988).

23. Mochalin, V. N., Shenderova, O., Ho, D. \& Gogotsi, Y. The properties and applications of nanodiamonds. Nat. Nanotechnol. 7, 11-23, DOI: 10.1038/nnano.2011.209 (2012).

24. Ivanov, M. G., Pavlyshko, S. V., Ivanov, D. M., Petrov, I. \& Shenderova, O. Synergistic compositions of colloidal nanodiamond as lubricant-additive. J. Vac. Sci. \& Technol. B 28, 869-877, DOI: 10.1116/1.3478245 (2010). https: //doi.org/10.1116/1.3478245.

25. Huber, R. C. et al. Extreme condition nanocarbon formation under air and argon atmospheres during detonation of composition b-3. Carbon 126, 289-298, DOI: 10.1016/j.carbon.2017.10.008 (2018).

26. Bagge-Hansen, M. et al. Detonation synthesis of carbon nano-onions via liquid carbon condensation. Nat Commun 10, 3819, DOI: 10.1038/s41467-019-11666-z (2019).

27. Chen, P., Huang, F. \& Yun, S. Characterization of the condensed carbon in detonation soot. Carbon 41, 2093-2099, DOI: 10.1016/s0008-6223(03)00229-x (2003).

28. Chen, P., Huang, F. \& Yun, S. Optical characterization of nanocarbon phases in detonation soot and shocked graphite. Diam. Relat. Mater. 15, 1400-1404, DOI: 10.1016/j.diamond.2005.10.008 (2006).

29. Pantea, D., Brochu, S., Thiboutot, S., Ampleman, G. \& Scholz, G. A morphological investigation of soot produced by the detonation of munitions. Chemosphere 65, 821-31, DOI: 10.1016/j.chemosphere.2006.03.027 (2006).

30. Deal, W. E. Measurement of chapman-jouguet pressure for explosives. The J. Chem. Phys. 27, 796-800, DOI: 10.1063/1. 1743831 (1957).

31. Coe, J. D. \& Margevicius, M. A. A new multiphase equation of state for composition b. Report LA-UR-16-25562 (2016).

32. Menikoff, R. Detonation waves in pbx 9501. Combust. Theory Model. 10, 1003-1021, DOI: 10.1080/13647830600851754 (2006).

33. Fried, L. \& Souers, P. Cheetah: A next generation thermochemical code. Report UCRL-ID-117240, Lawrence Livermore National Laboratory (1995).

34. Viecelli, J. A., Bastea, S., Glosli, J. N. \& Ree, F. H. Phase transformations of nanometer size carbon particles in shocked hydrocarbons and explosives. The J. Chem. Phys. 115, 2730, DOI: 10.1063/1.1386418 (2001).

35. Osswald, S., Mochalin, V. N., Havel, M., Yushin, G. \& Gogotsi, Y. Phonon confinement effects in the raman spectrum of nanodiamond. Phys. Rev. B 80, DOI: 10.1103/PhysRevB.80.075419 (2009).

36. Beaucage, G., Ulibarri, T. A., Black, E. P. \& Schaefer, D. W. Multiple Size Scale Structures in Silica-Siloxane Composites Studied by Small-Angle Scattering, chap. 9, 97-111. https://pubs.acs.org/doi/pdf/10.1021/bk-1995-0585.ch009.

37. Schmidt, P. W. Small-angle scattering studies of disordered, porous and fractal systems. J. Appl. Cryst. 24, 414-435 (1991).

38. Beaucage, G. Small-angle scattering from polymeric mass fractals of arbitrary mass-fractal dimension. J. Appl. Cryst 29, 134-146 (1996). 
39. Fratzl, P., Vogl, G. \& Klaumunzer, S. Small-angle scattering from porous amorphous substances. J. Appl. Cryst 24, 588-592 (1991).

40. Kratky, O. \& Porod, G. Diffuse small-angle scattering of x-rays in colloid systems. J. Coll Sci Imp U Tok 4, 35-70 (1949).

41. Rieker, T. P., Hindermann-Bischoff, M. \& Ehrburger-Dolle, F. Small-angle x-ray scattering study of the morphology of carbon black mass fractal aggregates in polymeric composites. Langmuir 16, 5588-5592 (2000).

42. Ferraro, G., Fratini, E., Rausa, R., Fiaschi, P. \& Baglioni, P. Multiscale characterization of some commercial carbon blacks and diesel engine soot. Energy Fuels 30, 9859-9866, DOI: 10.1021/acs.energyfuels.6b01740 (2016).

43. Sadezky, A., Muckenhuber, H., Grothe, H., Niessner, R. \& Pöschl, U. Raman microspectroscopy of soot and related carbonaceous materials: Spectral analysis and structural information. Carbon 43, 1731-1742, DOI: 10.1016/j.carbon.2005. 02.018 (2005).

44. Ferrari, A. C. Raman spectroscopy of graphene and graphite: Disorder, electron-phonon coupling, doping and nonadiabatic effects. Solid State Commun. 143, 47-57, DOI: 10.1016/j.ssc.2007.03.052 (2007).

45. Sze, S.-K., Siddeque, N., Sloan, J. \& Escribano, R. Raman spectroscopic characterization of carbonaceous aerosols. Atmospheric Envrionment 35, 561-568 (2001).

46. Bacsa, W. S., Lannin, J. S., Pappas, D. L. \& Cuomo, J. J. Raman scattering of laser-deposited amorphous carbon. Phys. Rev. B 47, 10931-10934, DOI: 10.1103/PhysRevB.47.10931 (1993).

47. Xie, F. Y. et al. Surface characterization on graphitization of nanodiamond powder annealed in nitrogen ambient. Surf. Interface Analysis 42, 1514-1518, DOI: 10.1002/sia.3350 (2010).

48. Zeppilli, S., Arnault, J. C., Gesset, C., Bergonzo, P. \& Polini, R. Thermal stability and surface modifications of detonation diamond nanoparticles studied with x-ray photoelectron spectroscopy. Diam. Relat. Mater. 19, 846-853, DOI: 10.1016/j.diamond.2010.02.005 (2010).

49. Robertson, J. Diamond-like amorphous carbon. Mater. Sci. Eng. R: Reports 37, 129 - 281, DOI: https://doi.org/10.1016/ S0927-796X(02)00005-0 (2002).

50. Mérel, P., Tabbal, M., Chaker, M., Moisa, S. \& Margot, J. Direct evaluation of the sp3 content in diamond-like-carbon films by xps. Appl. Surf. Sci. 136, 105 - 110, DOI: https://doi.org/10.1016/S0169-4332(98)00319-5 (1998).

51. Watkins, E. B. et al. Dynamic measurements of solid carbon cluster growth and morphology of high explosive detonation products. Sixt. Int. Detonation Symp. 821-829 (2018).

52. Schneider, C. A., Rasband, W. S. \& Eliceiri, K. W. Nih image to imagej: 25 years of image analysis. Nat Methods 9, 671-675, DOI: 10.1038/nmeth.2089 (2012).

\section{Acknowledgements}

We thank P. Bowden, A. Cartelli, E. Francois, L. Gibson, S. Jackson, and S. Vincent for shot assembly and detonation support. We also thank S. Doorn, D. Kelly and T. Williamson for instrumentation and/or sample analysis. And finally we thank D. Moore for his discussion and insights on detonation chemistry. Los Alamos authors acknowledge the support of the U.S. Department of Energy through the LANL/LDRD Program (LANL No. 20150050DR and 20200035DR). Use of the Stanford Synchrotron Radiation Lightsource, SLAC National Accelerator Laboratory, is supported by the U.S. Department of Energy, Office of Science, Office of Basic Energy Sciences under Contract No. DE-AC02-76SF00515. Los Alamos National Laboratory, an affirmative action equal opportunity employer, is operated by Los Alamos National Security, LLC, for the National Nuclear Security Administration of the U.S. Department of Energy under contract DE-AC52-06NA25396.

\section{Author contributions statement}

D.W.P. and D.M.D conceived the experiments. R.C.H. and D.W.P. conducted the experiments. R.C.H., A.C.A, K.J.G. and M.K.D analyzed the results. All authors reviewed the manuscript. 